\title{
Recent advances in chronic dialysis and renal transplantation in children
}

\author{
Robert H. Mak
}

Published online: 20 January 2009

(C) IPNA 2009

End-stage renal disease (ESRD) is a devastating chronic health problem in children, for which there is no life-long cure, not even after kidney transplantation. Fifty years ago, all children with ESRD died. The first pediatric dialysis programs were started in the 1960 s, and the first pediatric renal transplantation took place in the 1970s [1]. During the 1980 s there was a steady increase in numbers, and there has been relative stabilization since 2000. Now, almost all children with ESRD are treated with either long-term dialysis or renal transplantation. There has been an exponential increase in scientific literature in the field in the past two decades, particularly in the past 5 years (Fig. 1). Studies of outcomes over 20 years are now beginning to emerge $[1,2]$. Children with ESRD still have high mortality rates, estimated to be 30-times higher than expected for age compared with those of healthy children. On the other hand, the long-term survival of renal transplants has dramatically improved. Such information is important for pediatric nephrologists in counseling patients and families. However, it is always tricky to extrapolate past performance data to the future. The favorable impact of advances in technology and pharmacology as well as the wider acceptance of living-donor transplantation has to be balanced against the negative impact of new sets of sideeffects and the acceptance of more challenging patients, with severe and sometimes life-threatening co-morbidities, into the modern day dialysis and transplantation programs.

R. H. Mak $(\bowtie)$

Division of Pediatric Nephrology,

University of California, San Diego,

9500 Gilman Drive, Mailcode 0634,

La Jolla, CA 92093-0634, USA

e-mail: romak@ucsd.edu
In a series of teaching articles, we will summarize and integrate up-to-date clinical information and translate scientific discoveries into useful modules for application at the bedside. We aim to provide state-of-the-art summaries of the current knowledge and treatment guidelines for children with ESRD, which is also stage 5 chronic kidney disease (CKD). This is by no means an all-inclusive list, since many clinical and management issues are common to those in earlier stages of CKD and have been covered in an earlier series of teaching articles on CKD [3]. In each journal issue, there will be one article on long-term dialysis together with one renal transplantation article.

In the first issue, Drs. Shroff and Lederman will address "Long-term outcome of chronic dialysis in children" [4]. This article reviews the available outcome data for children on permanent dialysis as well as extrapolating data from larger experiences of dialysis in adults to inform pediatric nephrology practice. The multiple factors that may influence outcome, and, particularly, those that can potentially be modified, are discussed. Dr. Rees will address "Longterm outcome after renal transplantation in childhood" [5]. She will review factors influencing long-term outcome data after transplantation, overall patient survival and its factors, overall transplant survival and its factors, and final height and obesity, as well as psychosocial outcome. Transplantation is the treatment of choice for children with ESRD. It offers the best chances for growth, development and quality of life and is associated with a lower risk of death than is dialysis, particularly that due to cardiovascular disease. For these reasons, many pediatric nephrologists aim for preemptive transplantation for their patients, and more and more families are coming forward to be potential living donors for their children. This will further improve the overall transplant survival data in the future. 


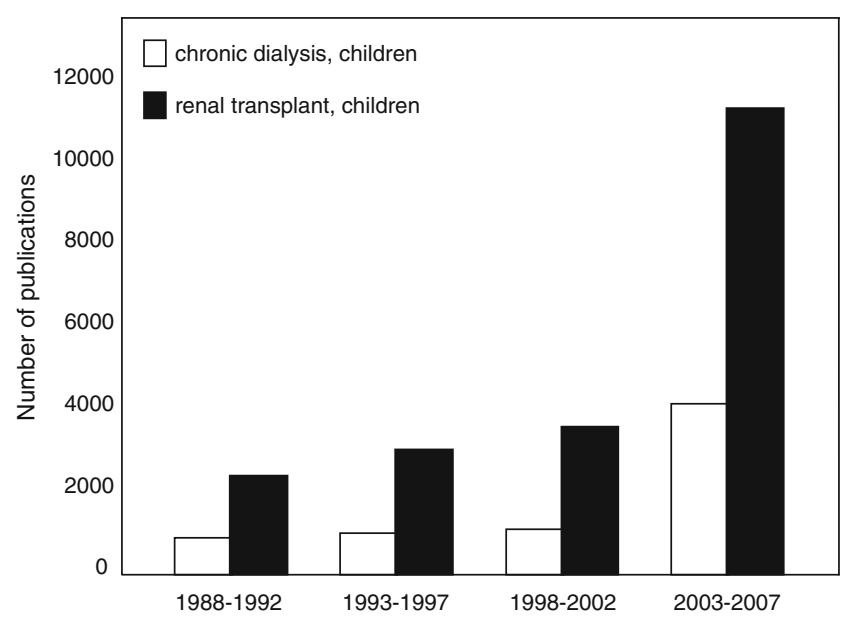

Fig. 1 Number of publications on long-term dialysis and renal transplantation in children, in 5-year periods over the past 20 years, obtained by searches in the PubMed database

Complications of ESRD contribute to long-term outcome, while technical difficulties remain a major challenge in treating children by long-term dialysis. Nutritional abnormalities in children on such dialysis, their management and their impact on mortality and morbidity rates will be addressed in two articles. Paglialonga and Edefonti will discuss the multidisciplinary approach to cachexia in patients on peritoneal dialysis in the article "Nutrition assessment and management in children on peritoneal dialysis" [6]. Drs. Srivaths, Wong and Goldstein will discuss nutritional status in children undergoing maintenance hemodialysis, with special focus on outcome, measurement of protein catabolic rate, and intradialytic parenteral nutrition, in the article "Nutritional aspects in children receiving maintenance hemodialysis: impact on outcome" [7]. Drs. Neu and Frankenfield will discuss the application of evidence-based dialysis adequacy guidelines in adult hemodialysis (HD) patients to observational data in 800 prevalent pediatric hemodialysis patients in the USA in order to identify pediatric HD patients at risk for poor outcomes in the article "Clinical outcomes in pediatric hemodialysis patients in the USA: lessons from CMS' ESRD CPM program" [8]. Although evidence-based dialysis adequacy guidelines are not yet available for use with children, current opinion-based guidelines will be addressed. Drs. Verrina, Cappelli and Perfumo will discuss "Selection of modalities, prescription and technical issues in children on peritoneal dialysis" [9]. Recent advances in kinetic modeling software programs, technology of new automated peritoneal dialysis (PD) machines, memorized delivery control and teledialysis are described. Drs. Fischbach and Warady will discuss, in the article "Peritoneal dialysis prescription in children: bedside principles for optimal practice" [10], how the understanding of the physiology of the peritoneal membrane, as a dynamic dialysis membrane with an exchange surface area recruitment capacity and unique permeability characteristics, results in the transition from an empirical prescription process based on clinical experience alone to the potential for a personalized prescription with individual adaptations. Drs. Chand, Valentini and Kamil contribute the article "Hemodialysis vascular access options in pediatrics: considerations for patients and practitioners" [11], in which they review the various types of pediatric vascular accesses used worldwide and the benefits and disadvantages of the various forms of access. Vascular access management requires proper advance planning, to assure that the best permanent access is placed, and a multidisciplinary team of nephrologists, nurses, surgeons and interventional radiologists, and ongoing monitoring, to ensure a long life of use. Drs. Honda and Warady will discuss "Long-term peritoneal dialysis and encapsulating peritoneal sclerosis in children" [12]. This complication is the most serious complication of long-term PD, with a mortality rate that exceeds $30 \%$, and it should be suspected in patients that have been on PD for more than 5 years with severe peritonitis, ultrafiltration failure, peritoneal calcifications, and persistently elevated levels of C-reactive protein. Finally, Drs. Chadha, Schaefer and Warady will discuss recent epidemiologic data from the International Pediatric Peritonitis Registry, in the context of designing individualized antibiotic therapy based on the patient, and center-specific history of microorganisms and their sensitivities, in the paper "Dialysis-associated peritonitis in children" [13].

Renal transplantation, although by no means a cure, is the treatment of choice for children with ESRD. However, many post-transplantation complications still remain as hurdles in long-term mortality and morbidity rates. These are discussed in a series of articles. Drs. Dharnidharka and Araya will discuss "post-transplant lymphoproliferative disease" [14], which usually involves uncontrolled B lymphocyte proliferation and straddles the border between infection and malignancy, since the Epstein-Barr virus (EBV) is intimately associated with the pathogenesis. Treatment strategies include reduction of immunosuppression, use of anti-B cell antibodies, infusion of EBV-specific cytotoxic $\mathrm{T}$ lymphocytes, and chemotherapy. Preventive strategies include serial EBV viral load monitoring, antiviral prophylaxis and pre-emptive immunosuppressive therapy. Drs. John and Kemper will discuss "urinary tract infections after renal transplantation" [15], which occur in $15-33 \%$ of patients. Risk factors include anatomical, functional and demographic factors as well as immunosuppression and presence of foreign material, such as catheters and stents. Meticulous surveillance, prompt diagnosis and treatment of urinary tract infections (UTIs) are important in minimizing acute morbidity and long-term graft dysfunc- 
tion. Dr. Seeman will discuss "hypertension after renal transplantation" [16], which ranges in prevalence from $60 \%$ to $90 \%$ depending on the method of blood pressure measurement and definition. It is an important risk factor in graft loss and morbidity and mortality rates in children with transplants. The most common causes are native kidneys, immunosuppressive therapy, renal graft artery stenosis and chronic allograft nephropathy. Control of hypertension leads to improved long-term graft and patient survival in adults and awaits confirmation in children. Drs. Harambat and Cochat will discuss "growth after renal transplantation" [17], which may be severely impaired. Short stature has major consequences for quality-of-life and self-esteem. The management of growth retardation in children after renal transplantation includes adequate nutritional intake, correction of metabolic acidosis, prevention of bone disease, steroid-sparing strategies and supraphysiological doses of growth hormone for selected patients. Drs. Fletcher, Nankivell and Alexander will discuss "chronic allograft nephropathy" [18], which is the leading cause of renal allograft loss in children after renal transplantation. The etiology involves immunological and non-immunological injury, including acute rejection episodes, hypoperfusion, ischemic reperfusion, calcineurin toxicity, infection and recurrent disease. Newer immunosuppression regimens, closer monitoring of the renal allograft and management of subclinical rejection may lead to reduced immune injury leading to chronic allograft nephropathy (CAN) but must be weighed against the risk of increased immunosuppression and calcineurin nephrotoxicity. Drs. Ying and Sarwal will discuss the development of microarray technology to improve early and non-invasive diagnosis of renal transplant dysfunction in an article "In praise of arrays" [19]. They will address the science as well as the application to clinical practice, including its limitations and recent developments in data analysis. In an article entitled "Mechanism of cellular rejection in transplantation" [20], Dr. Ingulli will discuss the in-depth understanding of the cellular mechanisms that result in both acute and chronic allograft rejection. These advances in basic science carry the promise of new strategies and targeted therapeutics capable of inducing long-lasting, allograft-specific tolerance.

It is important to note that the bulk of the current information and guidelines summarized in these teaching articles is mostly based on opinion and inconclusive evidence. It is also tricky to extrapolate past data into the future. The favorable impact of advances in technology and pharmacology as well as the wider acceptance of livingdonor transplantation has to be balanced against the negative impact of new sets of side-effects and the acceptance of more challenging patients, with severe and sometimes life-threatening co-morbidities, into the modern day dialysis and transplantation programs.

\section{References}

1. McDonald SP, Craig JC (2004) Long-term survival of children with end-stage renal disease. N Engl J Med 350:2654-2662

2. Ferres ME, Gipson DS, Kimmel PL, Eggers PW (2006) Trends in treatment and outcomes of survival of adolescents initiating endstage renal disease care in the United States of America. Pediatr Nephrol 21:1020-1026

3. Mak RH (2007) Chronic kidney disease in children: state of the art. Pediatr Nephrol 22:1687-1688

4. Shroff R, Ledermann S (2008) Long-term outcome of chronic dialysis in children. Pediatr Nephrol DOI:10.1007/s00467-007$0700-2$

5. Rees L (2007) Long-term outcome after renal transplantation in childhood. Pediatr Nephrol DOI:10.1007/s00467-007-0559-2

6. Paglialonga F, Edefonti A (2008) Nutrition assessment and management in children on peritoneal dialysis. Pediatr Nephrol DOI:10.1007/s00467-007-0719-4

7. Srivaths PR, Wong C, Goldstein SL (2008) Nutrition aspects in children receiving maintenance hemodialysis: impact on outcome. Pediatr Nephrol DOI:10.1007/s00467-007-0728-3

8. Neu AM, Frankenfield DL (2008) Clinical outcomes in pediatric hemodialysis patients in the USA: lessons from CMS' ESRD CPM Project. Pediatr Nephrol DOI:10.1007/ s00467-008-0831-0

9. Verrina E, Cappelli V, Perfumo F (2008) Selection of modalities, prescription, and technical issues in children on peritoneal dialysis. Pediatr Nephrol DOI:10.1007/s00467-008-0848-4

10. Fischbach M, Warady BA (2008) Peritoneal dialysis prescription in children: bedside principles for optimal practice. Pediatr Nephrol DOI:10.1007/s00467-008-0979-7

11. Chand DH, Valentini RP, Kamil ES (2008) Hemodialysis vascular access options in pediatrics: considerations for patients and practitioners. Pediatr Nephrol DOI:10.1007/s00467-008-0812-3

12. Honda M, Warady BA (2008) Long-term peritoneal dialysis and encapsulating peritoneal sclerosis in children. Pediatr Nephrol DOI:10.1007/s00467-008-0982-z

13. Chadha V, Schaefer FS, Warady BA (2009) Dialysis-associated peritonitis in children. Pediatr Nephrol DOI:10.1007/s00467-0081113-6

14. Dharnidharka VR, Araya CE (2007) Post-transplant lymphoproliferative disease. Pediatr Nephrol DOI:10.1007/s00467-007-0582-3

15. John U, Kemper MJ (2008) Urinary tract infections in children after renal transplantation. Pediatr Nephrol DOI:10.1007/s00467007-0690-0

16. Seeman T (2007) Hypertension after renal transplantation. Pediatr Nephrol DOI:10.1007/s00467-007-0627-7

17. Harambat J, Cochat P (2008) Growth after renal transplantation. Pediatr Nephrol DOI:10.1007/s00467-008-0787-0

18. Fletcher JT, Nankivell BJ, Alexander SI (2008) Chronic allograft nephropathy. Pediatr Nephrol DOI:10.1007/s00467-008-0869-z

19. Ying L, Sarwal M (2008) In praise of arrays. Pediatr Nephrol DOI: $10.1007 / \mathrm{s} 00467-008-0808-\mathrm{z}$

20. Ingulli E (2008) Mechanism of cellular rejection in transplantation. Pediatr Nephrol DOI:10.1007/s00467-008-1020-x 